\title{
Comparative Analysis of Three Crisis Intervention Models Applied to Law Enforcement First Responders During 9/11 and Hurricane Katrina
}

\author{
Cherie Castellano, MA, CSW, LPC, AAETS \\ Elizabeth Plionis, PhD
}

\begin{abstract}
Two distinct fields, crisis intervention (which targets civilian populations) and disaster mental health services (which targets first responders), have emerged in response to natural and man-made disasters. As a consequence of 9/11 and Hurricane Katrina, questions have been raised whether the occupational ecology of first responders has significantly changed. Two new concepts, the "high-risk rescuer" and the "rescuervictim," are identified. Using three field cases, this paper describes and analyzes the application of three different crisis intervention models for law enforcement first responders during 9/11 and Hurricane Katrina: (a) psychological first aid, (b) critical incident stress management, and (c) the Federal Emergency Management Association/Substance Abuse Crisis Counseling Program. Implications for meeting the mental health needs of first responders post-9/11 and -Hurricane Katrina are discussed. [Brief Treatment and Crisis Intervention 6:326-336 (2006)]
\end{abstract}

KEY WORDS: disaster mental health, law enforcement, first responders, occupational ecology, high-risk rescuers, rescuer-victim.

\section{The Issue}

Two distinct fields, crisis intervention (which targets civilian populations) and disaster mental health services (which targets first responders), have emerged in response to natural and manmade disasters. The degree to which concepts

From the Cop-to-Cop Crisis Intervention Hotline, University Behavioral Health Care University of Medicine and Dentistry of New Jersey, Piscataway, NJ (Castellano) and the Catholic University of America, Washington, DC (Plionis).

Contact author: Cherie Castellano, Director, Cop-to-Cop Crisis Intervention Hotline, University of Medicine and Dentistry of New Jersey. E-mail: ccaste17@aol.com.

doi:10.1093/brief-treatment/mhl008

Advance Access publication September 25, 2006 developed in one field can be applied to the other field, though controversial, has led to an array of training programs and a "tool kit" of interventions and crisis phase-specific responses. However, unlike civilian populations, first responders are affected by the dynamics of their organizational culture as well as the ecology of their occupation. Organizational dynamics have always influenced how mental health services are delivered and received.

Since $9 / 11$ and Hurricane Katrina, mental health professionals have become aware of two unique subgroups: (a) those first responders who are at higher than normal risk due to terrorist activities and (b) those first responders

(C) The Author 2006. Published by Oxford University Press. All rights reserved. For permissions, please e-mail: journals.permissions@oxfordjournals.org. 
who are simultaneously rescuer-victim as in Hurricane Katrina. First responders incur a higher risk of death and catastrophic injury when responding to terrorist incidents. During Hurricane Katrina, first responders faced ethical dilemmas and personal, family-related crises. Implications for meeting the mental health needs of these unique responder subgroups are explored by examining the three crisis intervention models identified in the study.

\section{Historical Perspective}

The field of psychological crisis intervention has existed since the early 1900s. It has developed concepts and practices that target civilian populations and those placed in harms way (the military). The development of disaster mental health (which targets first responders) as a field of practice developed in the early 1990s. Its primary target population is first responders. The development of this second field of practice was largely due to four factors: (a) the recognition of the occupational risk incurred by first responders, (b) the development of critical incident stress management (CISM) teams targeting emergency services personnel, (c) the expansion of the Salvation Army's disaster services to include disaster mental health personnel, and (d) the rise in global terrorism.

\section{First Responder Culture}

Historically, first responder personnel have been resistant to seeking mental/behavioral health services. Tensions have always existed between administrative and rank and file personnel. The public safety culture has been typified as one wherein seeking mental health services may be interpreted as a sign of "weakness." Furthermore, the culture attracts individuals who exhibit a "take charge, control-oriented style." First responders are commonly more com- fortable dealing with the problems of others rather than with their own problems. Establishing the trust needed for mental health counseling within such an environment is essential yet difficult for those mental health professionals seen as "outsiders."

It is no coincidence, therefore, that psychological support programs developed to serve the Secret Service; Fire Department of New York; New York Police Department; the Bureau of Alcohol, Tobacco, Firearms, and Explosives; and the National Fraternal Order of Police all utilize "peer support" (the use of line personnel specially trained in crisis intervention) models (Sheehan, Everly, \& Langlieb, 2004). Nonpeer mental health professionals often play an indirect or liaison role, with peer personnel taking the intervention lead. In the three field cases to be described below, the peer-to-peer counseling model was overwhelmed by the magnitude and unique characteristics of 9/11 and Hurricane Katrina.

\section{A Changing Occupational Ecology}

In recent years, first responders have been called upon to intervene in such disasters as the Oklahoma City bombing, the terrorist attack on the USS Cole, and the attacks of September 11, 2001. These disasters have added "terrorist attacks" to the typology of events to which law enforcement personnel respond. This has led to the realization that those who respond to terrorist attacks may themselves be targets of terrorist activities and are themselves at high risk for death or catastrophic injury.

In addition, several recent natural events, such as hurricanes Andrew, Katrina, and Rita, have led to the recognition that some responders fall into a category of rescuer-victim. Some first responders face ethical and personal crises when they and their families are themselves victims of a natural disaster. These recent events have led to increased awareness among 
mental health professionals of the changing occupational mental health risks encountered by first responders.

\section{Risk and Resources for First Responders}

\section{Risk}

It is well documented that public safety professions are at greater risk for the development of posttraumatic distress and alcohol abuse. Some personnel may present with depression/suicide or domestic violence. Whereas the general population suffers from post traumatic stress disorder (PTST) at a point prevalence of about 3\%, the law enforcement profession has a prevalence of around $10 \%$ and firefighters a prevalence between $10 \%$ and $30 \%$. Those exposed directly to a mass disaster are reported to have a PTSD prevalence of about 34\% (North et al., 1999). Although studies vary in their prevalence statistics, they seem to agree that there is a relationship between an individual's duration of exposure to traumatic imagery and the development of posttraumatic distress (Everly \& Mitchell, 1999).

Several studies (Bacharach \& Zelco, 2004; Institute of Medicine, 2003) have documented the mental health status of first responders following the terrorist attack of $9 / 11$. These studies suggest that public safety and emergency response professionals constitute a "high-risk" group with an increased probability of psychiatric morbidity, domestic violence, divorce, and shortened careers due to their occupational professional roles. In addition to their "normal" daily exposure to traumatic events, some responders are at heightened risk when they respond to terrorist events.

\section{Resources and Training Options}

Law enforcement personnel have access to mental health services sponsored by Employee
Assistance Programs (EAP), by departmental psychological services, by chaplain services, and by their unions. They also have access to traditional social services. However, because of the cultural dynamics of their occupation, law enforcement personnel tend to prefer models of mental health counseling that utilize peerto-peer counseling such as the Cop-to-Cop program.

\section{Evidence: Do Mental Health Services for First Responders Work?}

Little well-controlled disaster mental health outcome research for first responders exists (Institute of Medicine, 2003; National Institute for Mental Health [NIMH], 2002). Furthermore, law enforcement officers have several options to choose from when selecting to participate in mental health training. There are three major models of disaster mental health training targeting first responders: (a) Psychological First Aid (PFA), (b) CISM, and (c) the FEMA/SAMSHA Crisis Counseling Program (CCP). Is one model more effective than another?

\section{Comparison of Three Crisis Intervention Models Post-9/11 and -Hurricane Katrina}

\section{Psychological First Aid}

Description of the Model. Both the preexisting organizational culture of emergency response personnel and the changing occupational ecology of first responders must be taken into account when designing services that will meet the mental health needs of this population. As a model of practice, PFA has been endorsed by the Institute of Medicine (2003). The goals of this model are to provide (a) information and education, (b) comfort and peer support, (c) acceleration of recovery, (d) promotion of 
resiliency and mental health, and (e) access to continued care. This model of crisis intervention may be thought of as a form of "emotional first aid."

The New Jersey State Police (NJSP) EAP was developed in 1981 to provide the enlisted personnel of the State Police a confidential place to discuss any personal or work-related issues with a trained professional. Over the years, the office has become increasingly involved in CISM consultation and organizational training. A variety of adaptations to an early critical incident response model developed by Everly and Mitchell (1999) were necessary and were implemented in a model PFA response for first responders within the New Jersey Task Force One (NJTF-1)-Urban Search and Rescue Team.

Field Application of the PFA Model: World Trade Center. NJTF-1 is a volunteer Urban Search and Rescue Team comprising approximately 180 members, authorized and funded by the NJSP, Office of Emergency Management. On September 11, 2001, the NJTF-1 responded to the World Trade Center site and provided rescue and recovery efforts $24 \mathrm{hr}$ a day in 12-hr shifts for a 10-day period before returning "home" to Lakehurst naval base.

At the time of deployment, NJTF-1 was prepared with excellent rescue and recovery training, comprehensive equipment, and the capacity to live at the staging area. NJTF-1 was a self-contained unit. Team members had all the food, clothing, protective equipment, communications equipment, and shelter they would need for their 10-day stay. Team members were briefed to anticipate any and all needs created by the intense exposure related to the rescue and recovery mission. They erected tents and utilized decontamination trailers for showers when they returned from their 12-hr shift on "the pile." During every shift change, each team member was medically examined and cleared for further deployment if appropriate. What had not been anticipated, however, was the dramatic emotional impact this 10-day period would have on members of this unit.

Intervention Phases of the PFA. Fortunately for the NJTF-1 team members, the NJSP EAP utilized the basic tenants of PFA (developed for civilians) to respond to the psychological needs of this responder group. Developed by Everly and Flynn (2005), this model consists of five steps: (a) assessment phase, (b) stabilization phase, (c) triage phase, (d) communication phase, and (e) the follow-up or connection phase.

The PFA Assessment Phase. The first task of this phase is to provide immediate access to mental health assessment for those perceived as at high mental health risk because of the nature of the crisis event. To facilitate this phase, a mental health tent was placed at the rescue and recovery staging area to allow responders immediate access to care. The tent was staffed 24-7 with peers and clinical staff specifically trained to work with first responders dealing with critical incidents. The use of peer counselors fit the culture of law enforcement emergency personnel and lent credibility and familiarity to the counseling effort. Unlike civilians, law enforcement emergency personnel must continue to work professionally within the crisis environment to complete their mission.

The PFA Stabilization Phase. Ground zero was one of the most devastating rescue and recovery missions in the history of the United States. Itself volatile, the situation proved frustrating to the newly trained NJTF-1 team members. Three interventions proved beneficial during this phase. First, routine psychological checkups were initiated to follow medical checkups every $12 \mathrm{hr}$. Supportive and nonintrusive 
interventions were administered as warranted. Second, ongoing spiritual leadership, present from day one, sustained morale and proved to be a stabilizing force. A third stabilizing force came from the information provided by the media. Televisions were rigged up outside the tents and were watched regularly by NJTF1 team members.

The PFA Triage Phase. During this phase, job performance was assessed hourly. The NJSP EAP leaders communicated with the incident command structure, NJTF-1 team leaders, and peers regarding each member's ability to provide his/her special skill. Opportunities for "regrouping" a team were available should a NJTF-1 team member appear distressed and be in need of further evaluation and/or treatment. Group cohesion fostered a supportive environment and promoted individual resiliency. An unanticipated experience was the appearance of a barrage of volunteers and crisis counselors from all over the country eager to engage these workers in dialogue about their experiences.

The PFA Interactive Communication Phase. During this phase, members of NJSP EAP "joined" the responders by living in a tent in the staging area, wearing team fatigues, dining with the team members, and creating a daily religious service. Normalization was achieved by providing materials about critical incident stress. A nationally renowned speaker on law enforcement psychology spoke at a farewell ceremony as the team departed ground zero. The "farewell ceremony" was intended to prepare the NJTF-1 team members with education, awareness, and spiritual grounding. The ceremony also prepared members to meet and greet their families at the naval base later that day.

The PFA Hotline Phase. Initiating instant contact between team members, peer counselors, and nonpeer mental health professionals was a key component of this phase. The hotline also was a means of maintaining contact as part of the follow-up after termination. Within the first few days of deployment, a crisis helpline was created. Its goal was to ensure a rapid response following contact. Callers received further assessment and treatment as needed. This crisis helpline for the NJTF-1 team members remained active for several years post-9/11. Many rescuers utilized the line for resources and services for themselves and their families long after the PFA had been completed.

Summary. PFA is an acute mental health intervention with five phases. Its application to law enforcement emergency personnel deployed to ground zero for a 10-day mission is regarded as successful by those involved with it.

\section{CISM Model}

Description of the Model. The core elements of CISM are most frequently attributed to Everly and Mitchell (1997, 1999). However, it is important to note that the Salvation Army, Episcopal Church, American Red Cross, and the National Organization for Victim Assistance have utilized, with significant success, variations on the theme of multicomponent crisis intervention.

The model is consistent with Millon's three concepts of potentiating pairings, catalytic sequences, and the polythetic nature of a crisis (Millon, Grossman, Meagher, Millon, \& Everly, 1999). Potentiating pairings refer to the use of interacting combinations of interventions to achieve an enhanced clinical effect. Catalytic sequences refer to the sequential combination of tactical interventions to maximize their clinical effectiveness. The polythetic nature of the approach refers to the selection of tactical interventions as determined by the specific needs of each crisis situation. In other words, specific crisis interventions within the CISM 
model are to be combined and sequenced in such a manner so as to yield the most efficient and effective crisis intervention possible. The various combinations and permutations that are actually utilized within such an approach will be determined by the specific needs of each critical incident or traumatic event as they uniquely arise (Castellano, Everly, \& Langlieb, 2005).

Field Application. One of the many challenges faced by mental health personnel is to provide crisis mental health intervention to first responders in a chaotic and unpredictable event. Rescue and Recovery Teams from The New Jersey Port Authority Police Department (PAPD) were deployed to ground zero. Compared to previous crisis events, first responders suffered the largest loss of life in a single critical incident $(9 / 11)$ in the history of this country.

The number of first responders from the New Jersey PAPD who themselves died (37) on 9/11 as a consequence of responding to the crisis was unique. In addition, the duration of the rescue and recovery tours of teams exceeded normal tours of duty; most served in excess of 8 months. The duration of the rescue and recovery efforts provided a surreal world for first responders, sustained and punctuated with color-coded high alerts and threats of reoccurrence.

Intervention Model: CISM. On September 11, 2001, the cop-to-cop program (a mental health program established by the New Jersey state legislature for law enforcement personnel) experienced a $300 \%$ increase in calls; many from the New Jersey PAPD. In everyday crisis or critical incidents, peer support is often sufficient to meet first responders' trauma and stress management needs. The unprecedented events of 9/11 overwhelmed the peer-to-peer program, necessitating a collaborative approach between nonpeer professionals and peer counselors. As a consequence, the multicomponent CISM model was utilized to augment existing peer-to-peer counseling. Its six components are described below.

Component One: Acute Crisis Counseling Provided by Peer Counselors. Peer counselors (law enforcement officers who had received specialized training in psychological crisis intervention) were deployed from several locations to support first responders at ground zero. Nonpeer mental health counselors were initially used as "mental health backup" by the peer group leading the psychological crisis intervention initiative. During this 3-month phase, standard psychological crisis intervention techniques were used; individuals were assessed to determine their needs, and acute, one-on-one psychological crisis interventions were offered as needed (Everly \& Mitchell, 1999). Strategic planning, a collaborative effort between professional mental health counselors and peertrained crisis counselors, led to the development of other model components.

Component Two: Executive Leadership Program. Management and senior staff at PAPD requested additional assistance in providing crisis intervention for their officers with a specialized postimpact law enforcement program. A task force was convened to establish and deliver a program designed to educate and orient the participants on how to provide additional service. The leadership program recognized the importance of addressing the superior officers as a means of having them perceive behavioral health care services separate and distinct from their organizational structure. Attendees included medical personnel, mental health practitioners, public safety leaders, and administrators.

This leadership program consisted of a 2-hr lecture. Staff were given the opportunity to engage the participants. The question that had the greatest heuristic value in designing 
subsequent interventions was "what do you think you need and what will help you?" The perceived success of this leadership training prompted a continuation of services as part of a "trickle down" effect. Over 100 Acute Traumatic Stress Management Training sessions were provided in multiple commands. The success of such trainings depended on whether the recipient group perceived the training as accessible and accommodating rather than cumbersome and interfering.

Component Three: The Multidisciplinary Team. A multidisciplinary team worked 24-7 to assess the status of those working at ground zero. Members of the team provided customized training sessions to meet the unique needs of first responders as they were identified. Because members of law enforcement tend to avoid anything individually focused or considered "psychiatric," or even "therapeutic," group crisis interventions were utilized and were conceptualized as "training." Interventions were more likely to be well received and utilized if presented and perceived as training.

Component Four: Acute Traumatic Stress Group Training Sessions. These sessions consisted of a 2-day psychoeducational group format. The group format (rather than a focus on individual interventions) was seen as an important addition. The group was seen as both logistically efficient and better able to normalize reactions, build informal support networks, and teach practical coping skills. The group format also proved to be a valuable triage tool. Most importantly, the group countered the sense of social alienation that often accompanies posttraumatic distress. Outside experts spoke on law enforcement personality traits, the signs and symptoms of stress and distress, and common effective coping mechanisms. One special challenge was the difficult task in processing survivor guilt and grief among those deployed to 9/11.
Although the intent of the group sessions was education and training, feedback from the facilitators indicated that most groups manifested a therapeutic quality. Officers described our sessions as the first supportive group experience they had where they could discuss the traumatic events associated with the 9/11 disaster.

Component Five: Hotline. Prior to September 11,2001 , the law enforcement hotline was created. Subsequent to September 11, the hotline served as an invaluable tool for passive outreach and for active feedback from field personnel. The line operated 24-7. Data collected from initial calls were used to create the hotline manual and develop resources.

Component Six: Reentry Program. A reentry program was initiated to meet the needs of high-risk rescuers. The mental health literature recognizes that prolonged exposure to danger, death, and devastation creates difficulties as members return to routine tasks on the job and family life. The intent of the reentry program was to prepare participants for a return to routine job functions and normal family life.

Summary of the CISM Model. The CISM model described above utilized a combined large group, small group, family, and individual intervention approach to engage participants who historically are viewed as intervention resistant. Each intervention format offered unique advantages that collectively served as a form of continuum of care, lacking when only one intervention format is used. The components in this application of CISM were formulated only after listening to the needs of the officers and carefully reviewing relevant literature within the fields of counterterrorism, law enforcement stress, psychological trauma, peer support, CISM, "debriefing," and suicide prevention. 


\section{The FEMA/SAMSHA CCP Model}

Description of the CCP Model. This model has been frequently used for natural disaster response. The CCP model assesses strengths, seeks to restore predisaster functioning, accepts content at face value, validates common reactions, and has a psychoeducational focus.

Field Application. The CCP model was adapted for first responders on the Gulf coast in response to Hurricane Katrina. A unique aspect of this crisis event was that law enforcement professionals became simultaneously rescuer and victim. Unlike most other disasters, the families of these first responders were themselves endangered by the disaster.

Three critical incidents resulted from Hurricane Katrina. The first was the actual natural disaster. The storm and the subsequent breach of the levees caused massive flooding over $80 \%$ of the city. The second involved the breakdown of local, regional, state, and national (FEMA) emergency response organizations. The third critical incident involved the unprecedented personal and ethical crises faced by law enforcement personnel during the hurricane.

Assess Strengths. The first task of the CCP model is to assess strengths. Levees separating Lake Pontchartrain from New Orleans were breached by the storm surge, ultimately flooding about $80 \%$ of the city. According to estimates, Katrina caused damages in excess of $\$ 75$ billion. Shortly after the hurricane ended on August 30, residents who did not or could not leave New Orleans began looting stores. Many were in search of food and water, though some stole nonessential items as well. Reports of carjackings, murders, thefts, and rapes flooded the news; many of these reports were later determined to be rumors. Emergency personnel lacked the ability to secure the scene; geographically, few areas of high ground existed, and facilities and equipment were flooded. Individ- uals who could help other individuals often did so. Outside resources (material and human) were needed as the crisis unfolded; however, requests for such help were delayed. When available, resources could not readily be deployed because of the extent of flooding.

Organizational Breakdown. The storm and subsequent levee breaks disrupted regular police functions. Members of the police force were relieved from their shift and told to stand by within the city. Many of them found rooms in vacant hotels, with friends or in their homes. They were told to return at 4:00 p.m. Before they could do so, they found themselves flooded and marooned. One officer swam with her 8-month-old baby to "headquarters" that was by then an abandoned catering facility, the highest ground in the district. Water continued to rise rapidly despite the storm's end.

The same forces of nature, which prevented local emergency transportation and communication, disseminated law enforcement leadership and created an organizational crisis; one that bubbled up from local to regional to state and to national emergency response commands. Communication failed as police radios, cell phones, and all landlines went out. No central command existed. What facilities and equipment did exist were improvised. Standard operating procedures became moot.

Restore Predisaster Functioning. Chaos and Reorganization. Law enforcement attempted to regroup by forming Krewes (a Mardi Gras term referring to the custom of a club or group creating a float). Krewes operated independently. Krewes were the only option law enforcement personnel had to both function and survive. Although Krewes generally grew closer, some fracture lines occurred between different shifts, between superior officers and rank and file police members, and between those officers who had left to take care of their 
families (even though many ultimately returned) and those who stayed on the job. It was impossible to restore predisaster functioning while $80 \%$ of the city remained flooded. Unlike other crises, law enforcement was unable to secure the scene.

Acceptance and Validation. Law enforcement personnel themselves lacked basic safety, housing, a command structure and facility, clean clothes, food, and water. Rescuers themselves felt abandoned.

It is estimated that over $80 \%$ of the officers surveyed had lost their homes and all their possessions. Members of their families became ill, suffered injuries, and were relocated. Friends and family members were lost.

In addition, members of the New Orleans police department faced personal and ethical crises. Law enforcement members had to decide whether to stay on the job in a spirit of loyalty to the brotherhood and their oath to protect the public or rescue their own family members who were themselves endangered by Katrina. In retrospect, mental health practitioners recognize that members of the New Orleans Police Department were simultaneously rescuer and victim. Unlike the media support the first responders felt at 9/11, media coverage of Katrina further demoralized the members of law enforcement by depicting them as malfeasant.

Ethical dilemmas. Unlike other critical incident events, law enforcement personnel faced a basic conflict between their sworn oath to protect and serve the public and their personal commitment to protect and save their families. One officer described his dilemma as a conflict between his oath as a police officer and his vows of marriage and his obligations as a parent to protect his family. Law enforcement personnel were not prepared for the ethical dilemma they faced. Mental health professionals recognize that recovering from a "no-win" critical incident is more complicated.
Psychoeducation. Written materials and roll call presentations informed officers about "normal reactions to abnormal events." Law enforcement personnel were connected to available resources in the outreach phase of the $\mathrm{CCP} /$ FEMA model. Small group meetings were held at each precinct. Interventions were limited to recognition of the problems incurred and validation of the rescuer-victim experience. As was true at 9/11, some members benefited from faith-based or spiritual discussions. Topics included forgiveness (of self and others).

\section{Summary of the FEMA/SAMSHA CCP} Model. The unusual circumstances of Hurricane Katrina rendered the implementation of this model difficult. Whenever possible, individual officers sought one-on-one peer crisis counseling. Some diffusings (group format) occurred where and when feasible. Stabilization was less effective given the unique features of this crisis. Small gestures such as garbage removal from a temporary command post or the provision of clean shirts were regarded as a show of support.

\section{Implications for Practice}

\section{Psychological First Aid}

Providing mental health support at the staging area of a crisis (as in 9/11) is a strategy that benefits first responders by making access to support readily available and more likely to be used. A spiritual component to crisis intervention is beneficial to many first responders.

\section{The CISM Model}

Compared to other models, this model is fluid and offers a wide range of intervention options during each phase of a crisis. The Reentry program component developed in response to the Port Authority team assigned to ground zero 
proved helpful to those first responders who are deployed to a crisis site for long periods of time. In the case example cited, the use of psychoeducational support groups was regarded as a more effective intervention than one-on-one crisis counseling. Customizing intervention content based on input from first responders on what they need is more effective than offering predetermined content alone. Asking "what do you think you need?" or "what will help you now?" is effective in guiding the intervention focus. The multiple phases of a crisis intervention require specific skills at different times. When using a multicomponent CISM model, it is important that one singular entity controls and integrates the multiphase plan (Employee Assistance Professionals' Association, 2002; NIMH, 2002).

\section{The FEMA/SAMSHA CCP Model}

Because the geographic and physical facilities of first responders in New Orleans were demolished, it is important to adapt crisis intervention outreach and follow-up services to the functional community of first responders. It is important to redefine a geographic community as a functional "first responder community." Primarily a model used to intervene with civilian populations, the CCP model must take into account the unique organizational and occupational features of first responders. Analysis of its application in Hurricane Katrina led to increased awareness of the rescuer-victim and the ethical dilemma faced by first responders when their own families are themselves endangered by the disaster.

\section{Training Content}

Though training programs are intended to be proactive in preparing counselors to deal with the mental health needs of first responders, not all contingencies can be anticipated. Experience, unfortunately, is the only real educator.
The events reported in this paper have led to increased awareness of two new first responder subgroups, the high-risk responder and the rescuer-victim.

Other areas to be incorporated in training content include content on ethical decision making and ethical dilemmas. First responders must have a clear understanding of their value systems. Drills and contingencies must be incorporated to handle organizational breakdown and situations where the rescuers families are themselves endangered.

\section{Recovery}

What facilitates recovery? Some (Reese, 1987) argue that there is a role for anger in the recovery process. Others argue that denial plays a role in that it allows individuals to move on. From these three cases, we learn that recovery also depends on augmentation of peer-to-peer counseling programs through the use of nonpeer professionals in collaboration with peer counselors.

\section{Summary}

The field events reported in this paper suggest that providing disaster mental health services to first responders requires customized fluid crisis intervention services based on practical application rather than on bounded theory and training auspice (Castellano et al., 2005, p. 51). Selecting one model over another may compromise the practitioner's ability to offer crisis mental health intervention for first responders appropriate to the crisis event they work. Techniques that are appropriate for one phase may not be sufficient for subsequent phases of the event. The ability of a mental health practitioner to engage in best practice depends in part on his/her familiarity with a variety of crisis intervention models. More importantly, it depends on field data about what is and what is not working at the moment under different circumstances. 


\section{Acknowledgments}

Conflict of Interest: None declared.

\section{References}

Bacharach, S. B., \& Zelco, H. (2004). On the frontline: The work of first responders in a post 9/11 world. NY: Cornell.

Barkun, M. (April, 2002). September 11th through Conspiracists' Eyes. Paper presented to the Critical Incident Analysis Group (CIAG) conference, "Terrorism, Intelligence and Democracy," University of Virginia, Charlottesville. http://www. hsc.virginia.edu/csmhi/ciag/report_barkun.html

Castellano, C., Everly, G. S., \& Langlieb, A. (2005). Psychological counterterrorism and World War IV (p. 51). Ellicott City, MD: Chevron Publishing Company.

Dyregrov, A. (1998). Psychological debriefing: An effective method? Traumatology, 4(2).

Employee Assistance Professionals' Association. (2002, November). Employee assistance programs' critical incident management: Report of the EAPA Disaster Preparedness Task Force. Boston, MA: Author.
Everly, G., \& Flynn, B. (2005). Principles and practical procedures for acute psychological first aid for personnel without mental health. Journal of Emergency Mental Health, 8(2).

Everly, G. S., Jr., \& Mitchell, J. (1997). Critical incident stress management. Ellicott City, MD: Chevron.

Everly, G. S., Jr., \& Mitchell, J. (1999). Critical incident stress management (2nd ed.). Ellicott City, MD: Chevron.

Millon, T., Grossman, S., Meagher, S., Millon, C., \& Everly, G. S., Jr. (1999). Personality-guided therapy. NY: Wiley.

National Institute for Mental Health. (2002). Mental health and mass violence. Washington, DC: U.S. Government Printing Office.

North, C. S., Nixon, S. J., Shariat, S., Mallonee, S., McMillen, J. C., Spitznagel, E. L., et al. (1999). Psychiatric disorders among survivors of the Oklahoma City bombing. Journal of the American Medical Association, 282, 755-762.

Sheehan, D., Everly, G. S., Jr., \& Langlieb, A. (2004, September). Current best practices responding to major critical incidents. FBI Law Enforcement Bulletin, 1-11. 